\title{
Evaluating the Performance of a MAC Protocol in WANETs using Directional Antennas: A Survey
}

\author{
Nitesh Kaushik \\ Department of Computer \\ Science \\ Suresh Gyan Vihar University \\ Jaipur
}

\author{
Kavita, Ph.D. \\ Department of Computer \\ Science \\ Jagannath University \\ Jaipur
}

\author{
Narendra Singh Yadav, \\ Ph.D. \\ Department of Computer \\ Science
}

Sri Balaji College of Engg. and

Tech, Jaipur

\begin{abstract}
Reduction in radio interference \& improvement in communication throughput we can achieve by the use of directional antennas in wireless Adhoc network..Deafness \& new hidden terminal problem causes a degradation in performance, which is introduced by directional antennas in wireless media access control. To solve these problems we can propose some protocols like circular RTR directional mac protocol to solve the above problems. We can evaluate the results of these protocols by simulation.
\end{abstract}

\section{General Terms}

Switched Beam Antenna, Adaptive Array Antenna, RTS reception and CTS transmission

\section{Keywords}

Directional antenna, MAC protocol deafness, new hidden terminal, WANETs

\section{INTRODUCTION}

A wireless network of decentralized type with a gathering of wireless nodes is referred as Wireless Ad hoc Network (WANETs).Flexilibility, robustness and low cost are some of the main advantages of these networks. Its application areas are in military activities, disaster recovery and in emergency operations[5].The issues for example in MAC layer includes the hidden and the exposed terminal problem collision detection, which require new medium access algorithms[6-7].All nodes are equipped omni-directional antennas in wireless Ad hoc networks.

A collection of wireless nodes in a wireless network is referred as WANET's. These networks can self-organize dynamically into arbitrary or temporary network topologies without using any local infrastructure [3].Flexibility, low cost, and robustness are the main advantages of these networks. easily set up and enduring to natural catastrophes and wars are done by these networks. making it well met for wide applications, such as military activities, emergency operations and disaster recovery [5] are the characteristics of these networks. Example, issues of collision detection ,hidden and exposed terminal problems, demanding new medium access algorithms for MAC layer[6-7].Equipped nodes with Omni-directional antennas in WANET's ,example IEEE 802.11 MAC protocol[8]. However, the MAC protocols with Omni directional antennas consume a network capacity with a large portion reserving the area of wireless medium [9]. The use of directional antennas has been proposed to solve this problem, in which sender and receiver nodes mainly controls the transmitting and receiving beams towards each other only. These antennas improve the performance of the network, example, spatial reuse ranging gain on a higher side and reducing interference by radio beam directing on desired direction [6].Many MAC protocols use directional antennas for WANETs proposed [9-11], referred as Directional MAC (DMAC) protocols. Mainly the proposed DMAC protocols are adapting the use of IEEE802.11 over directional antennas [12].The application of IEEE 802.11 to WANETs with directional antennas, mainly, induces channel access problems of locationdependent carrier sensing, including three new hidden terminals and deafness.

The deafness problem arises due to beam- forming in directional antenna operations [13].The symmetry-gain new hidden terminal problem arises due to beam forming gain asymmetry in directional antennas, the unheard-RTS/CTS new hidden terminal problem arises because of directional operations [14], and the multichannel new hidden terminal problem occurs due to operations in multi-channel MAC protocols [15]. These problems may give results in severe performance degradation by packet drop and waste of network capacity.We also have some protocols such as circular RTR directional MAC protocol for WANET's for solving the deafness and hidden terminal problems. In CRDMAC protocol mainly utilizes directional transmissions and multi-channel separation techniques to increase the coverage area for wireless communication. It mainly includes RTS directional /CTS handshake ,the data transmission in the main data channel as well as to transmit the cast of Ready to Receive (RTR) messages in the control sub-channel. Basically it is based on a sub-channel for circular directional RTR messages, which check the neighbor nodes across the transmitter for intended communication.

\section{RELATED WORK}

The use of directional antennas has been studied over recent years. IEEE 802.11 DCF is based on the technique of MAC i.e. CSMA/CA by using Omni-directional antennas at physical layer. The RTS and CTS control packets reduce the hidden-terminal problem through the Network Allocation Vector (NAV) [8]. IEEE 802.11 DCF use the binary exponential back off, which multiplicatively decreases the transmission rate. IEEE 802.11 is a CSMA/CA protocol which mainly performs physical carrier sensing before starting transmissions [16]. on sensing the channel as idle for DIFS (DCF Inter- frame Spacing) duration, the protocol invokes a back off mechanism for the purpose of contention resolution.

\subsection{Directional Antennas classification}

They are different from Omni-directional antennas as we know. They are constructed to have certain favored transmission and reception directions. In WANETs, directional antennas, also called as smart antennas, consist of a number of radiating elements and a network control unit basically. Digital signal processor is being used for implementing control unit ,which is mainly the intelligence part of smart antennas. Smart antennas are mainly categorized as of two types [17-18]. 


\subsubsection{Switched Beam Antenna:}

In these, the antenna array is combined with a fixed Beam Forming Network (BFN), which mainly consists of a predetermined set of weight vec- tors. Based on the direction-of-arrival estimation (DoA), the BFN selects a weight vector to be applied to the signal transmission/reception by the antenna array. The antenna adaptively switches to one of the predefined set of beams in a desired direction. Switched beam antennas are providing most of the benefits of smart antennas with less complexity and expense.

\subsubsection{Adaptive Array Antenna:}

They are also known as the Steered Beam Antenna systems, providing a high degree of flexibility in configuring the radiation patterns. For signal transmission/reception and continuous tracking DoA algorithm is applied. The main lobe antenna array is directed towards the target using phase shifters, which constructs a steerable radiation pattern. In spite of that, the requirements are continuously locating and tracking signals to complicate the signal processing task, and result in significant increase in power consumption.In this paper, we mainly utilizes the switched beam antennas in the DMAC protocols for WANETs for energy efficiency.

\subsection{DMAC protocol}

Directional antennas needs to be controlled by upper layers of the networking protocol stack to realize the potentials of the beam forming systems [7]. The most important layer to be modified, is the MAC layer ,because it lies just above the physical layer. The aim of DMAC protocols is to set the rules for enabling efficient and fair sharing of the directional wireless channel in common. Recently, many protocols related to DMAC have been proposed, which mainly attempt to mitigate the basic issues in WANETs with directional antennas. Korakis et al. [19] mainly proposed a Circular RTS MAC. which sequentially transmits multiple RTS on all beams for each data frame for scanning the area around the transmitter. It offers improvement of Omni-directional transmissions, Although the performance was degrade by circular directional RTS. Jakllari et al. [20]protocol multiple directional frames were transmitted in a circular fashion for handling deafness on the receiver side.in his proposed circular RTS and MAC protocol. However increasing delay incurs large control overhead. Gossain et al. [21] proposes a multiple directional RTS messages which are transmitted through the beams, diametrically opposite directions with neighbors proposed a MAC protocol for directional antennas.

\subsection{Multi-channel MAC Protocols}

The MAC protocol is used for setting rules in order to have efficient and genuine sharing of the common wireless channels. The multi-radio multi-channel MAC protocol mainly increases the utilization of channel with as many communications as possible [16,21]. Mainly in many researches, multichannel MAC protocols have been proposed, which control separation techniques in multichannel radio systems [15,23-24].Variety in multichannel MAC provides various purposes and uses: intending some for enabling simultaneous transmission of data, reserving Some future data uses while the current data channel mainly is occupied, using separate signals for solving hidden terminal problem. Two sub-channels are obtained by separating one radio channel ,i.e.a data channel and control channel, by mainly employing the multichannel MAC protocol.

\section{OVERVIEW OF DEAFNESS AND HIDDEN TERMINAL PROBLEM}

In this, we introduce the four way handshake for channel reservation [7] in the MAC protocols. In this we describe the channel access problems of DMAC protocols in WANETs with directional antennas, the deafness problem $[10,13]$ and three hidden terminal problems [10,14-15,19]. For convenience, the directional RTS and the direction al CTS are called DRTS and DCTS for short in the following

\subsection{Four-Way Handshake and Channel Reservation}

In this technique for channel reservation and data transmission in wireless communications. It performs same to that of IEEE 802.11 standard [8] in the DMAC protocols, including RTS (Request To Send) transmission, CTS (Clear To Send) transmission, DATA exchange, and ACK (Acknowledgement) exchange. An idle node listen to the channel in the Omni mode From a particular direction ,on arriving a signal, on receiving, it was locked. In this we refers to $\mathrm{S}$, which is the sender node and $\mathrm{R}$, which is the receiver node $\mathrm{R}$ for describing the four-way handshake in the DMAC protocols.

\subsubsection{RTS Transmission}

To mainly detect, whether it is safe to transmit RTS to node $R$, node $S$ first performs physical carrier sensing using a beam form ${ }_{B} R$. If the channel is being sensed idle, the DMAC checks its Directional NAV (Network Allocation Vector) Table, which arranges a virtual carrier sense status for every DoA (Direction of Arrival). Once if a node $S$ finds out that it is safe to transmit using $B^{R}$, the DMAC at node $S$ requests the physical layer to transmit the RTS to node $R$ using beam $B^{R}$.

\subsubsection{RTS reception and CTS transmission}

After receiving the RTS from $S$, node $\mathrm{R}$ it determines the direction to send the CTS in response. If NAV table at $\mathrm{R}$ permits transmission in this direction, then node $\mathrm{R}$, DMAC request to beam form in this direction with beam BS for CTS .

\subsubsection{CTS Reception and DATA/ACK Exchange}

If the node $S$ receives the CTS, it basically initiates the transmission of DATA packets using beam $B^{R}$. On receiving data successfully transmits ACK by the use of beam BS. Basically the other neighbor nodes in the transmission range, receive the ACK packet, and modernize their NAV tables with the respective DoAs.

\subsection{Deafness}

The deafness problem in the DMAC protocols is explained from in Fig 1.assuming note A to be data packet from node B and all nodes are idle. The DMAC basically requires node $\mathrm{B}$ to beam form in the direction of node $\mathrm{A}$, and detecting the DIFS duration for the channel. But on finding the channel idle, node B proceed to the back off phase and mainly counts down the back off counter which still beam formed towards A. On checking node $\mathrm{Z}$ mean to communicate with node $B$, which is counting down its back off counter. If RTS to node B was not transmitted then node B will unable to obtain RTS in that direction. So in the absence of the response from node $\mathrm{B}$, node $\mathrm{X}$ would continuously back off and retransmitting RTS till the ending of dialog between node Band A. The unused retransmissions from node $\mathrm{X}$ are the outcome of deafness 


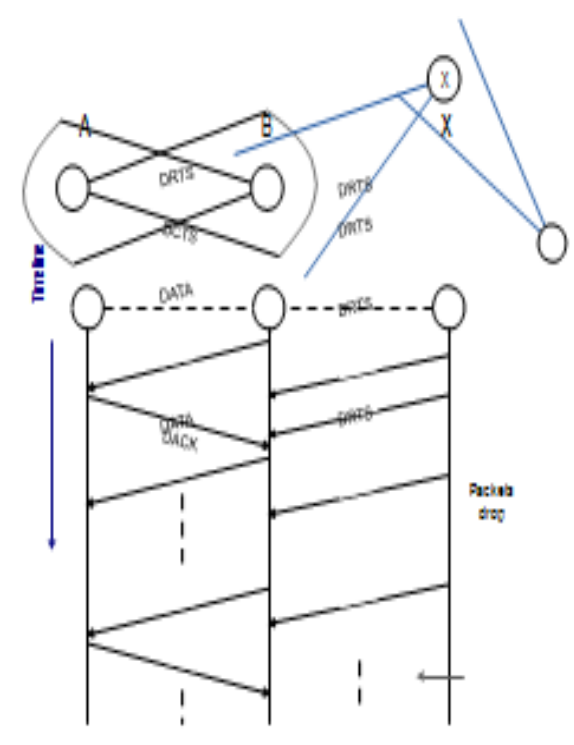

Fig 1 A scenario of deafness in DMAC protocols

Once node B has completed the transmitting of the first data message, it can immediately prepare for transmitting the next message by beam forming in the same direction mainly towards $\mathrm{A}$, and then can repeat the sequence of DMAC operations with $\mathrm{A}$. If the node $\mathrm{B}$ remains back logged for a very long time, node $\mathrm{X}$ can end up dropping. At that time only, continuously repeated failure to communicate to node B, may cause node X's contention window growing exponentially. If the node $\mathrm{X}$ has multiple packets in line for node B, transmits a RTS directional. Thus, the node $\mathrm{Y}$ can experience stretched deafness in the same way, until $\mathrm{X}$ has dropped all its packets. But on chain transmission of nodes, where any of the node would have interact successfully, evokes a new problem of, deadlock [13]. It is a serious problem of multi-hop transmission, where transmitter is the intended receiver of the node.

\subsection{New Hidden Terminals}

This problem in multi-hop wireless networks can be solved by using RTS/CTS control packets in the CSMA/CA of 802.11 DCF [8], which are mainly transmitted Omni directionally. Directional transmission of RTS/CTS in DMAC protocols starts of two types of new hidden terminals, the asymmetry-gain new hidden terminal and the unheard-RTS/CTS new hidden terminal. On discussion hidden terminal problem is another of new type related to multichannel MAC protocols the multi-channel new hidden terminal.

\subsubsection{New hidden terminal due to asymmetry in gain}

In this paper, we introduce the new type of hidden terminal problem related to packet collision in WANETs with directional antennas. We say it the asymmetry-gain new hidden terminal in this paper, and explain it using the following concept .Now consider the concept in Figure 2.we assume that all nodes in this scenario are idle, and nodes in the idle state have a gain of $G^{O}$ (Omni mode).we assume that node A transmits a DRTS to node $B$, and $B$ replies with a DCTS. M a in ly DATA transmission begins from node $A$ to $B$, and both the nodes are pointing their transmission and reception beams with the gain $G d$
Node $D$ unable to sense this transmission when this communication in progress, and we assume that node $D$ has packets to send to $C$. Node $D$ basically senses the channel with a directional beam pointed towards $C$ and concludes that the channel is mainly idle, and sends a DRTS to node $C$. So, since node $B$ is basically receiving DATA from $A$ in same direction, it is possible that the DRTS from $D$ interferes at node $B$. So, In other words, collisions can occur at the unanticipated receiver nodes which are in the range of same direction, when they basically transmit and receive with directional beam of $G^{d}$.

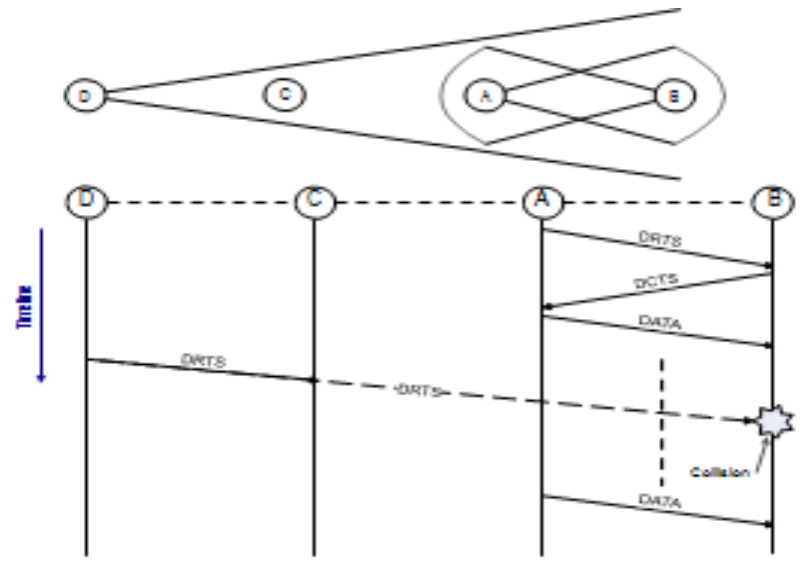

Fig 2 A scenario of asymmetry gain new hidden terminal in DMAC protocols

\subsubsection{New hidden terminal due to unheard RTS/CTS}

The second new hidden terminal, the un- heard-RTS/CTS new hidden terminal discussed in this paper. Consider the concept in Fig 3. Assume that the node $\mathrm{C}$ is communicating with node $\mathrm{D}$ with directional beams towards each other. when this transmission is in progress, node B is continuously transmitting packets A using directional antennas in the same time. when the communication between $\mathrm{B}$ and $\mathrm{A}$ in progress, we assume that the node $\mathrm{C}$ finishes the communication with $\mathrm{D}$ and now the node wants to transmit the packets to A. Node C's directional NAV table indicates that now it is allowed to transmit towards A and C finds the channel is idle on the performance of physical carrier sense. Thus, the node $\mathrm{C}$ continuously transmits the RTS, and a collision may occurs at node A.

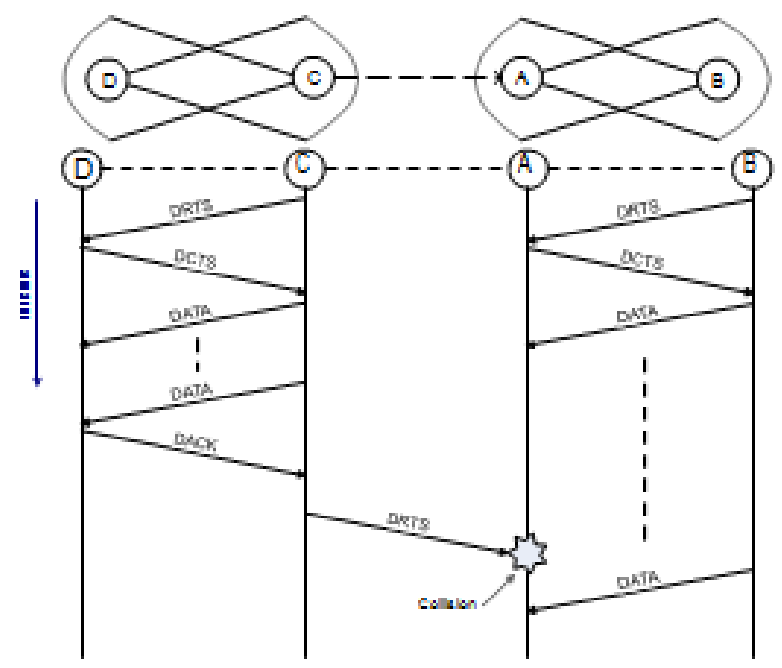

Fig 3 A scenario of unheard -RTS/CTS new hidden Terminal in DMAC protocols 


\subsubsection{New hidden terminal due to multi- channel collision}

Now we introduce the third new type of hidden terminal problem related to packet collision in multi channel environment using Omni antennas, which is referred as the multi-channel new hidden terminal in this paper. The traditional multi-channel MAC protocol, a node with a single transceiver, which is communicating in one channel, cannot hear packets transmitted in other channels .In the multi-channel new hidden terminal problem in multichannel MAC protocols using the following concept in Fig 4 , which have two radio channels, a control channel for RTS/CTS packets and a data channel for the data packets. Node B has packets for A, so B sends the RTS on Channel 1 , which is in the control channel. Node A gives the reply from a a CTS back to B, and choose the Channel 2 for data communication. However, when the node A sends the CTS to $\mathrm{B}$, node $\mathrm{C}$ was busy in transmitting DATA on another channel (channel 2) to $\mathrm{D}$, so it is unable to hear the CTS from A. so A is unable to know the receiving of DATA on Channel 2, C may be continuously communicating with $\mathrm{D}$, and will result in a collision at node A. so the multi-channel new hidden terminal occurs due to the nodes listening different channels, so it is difficult using virtual carrier sensing for avoiding hidden terminal problem.

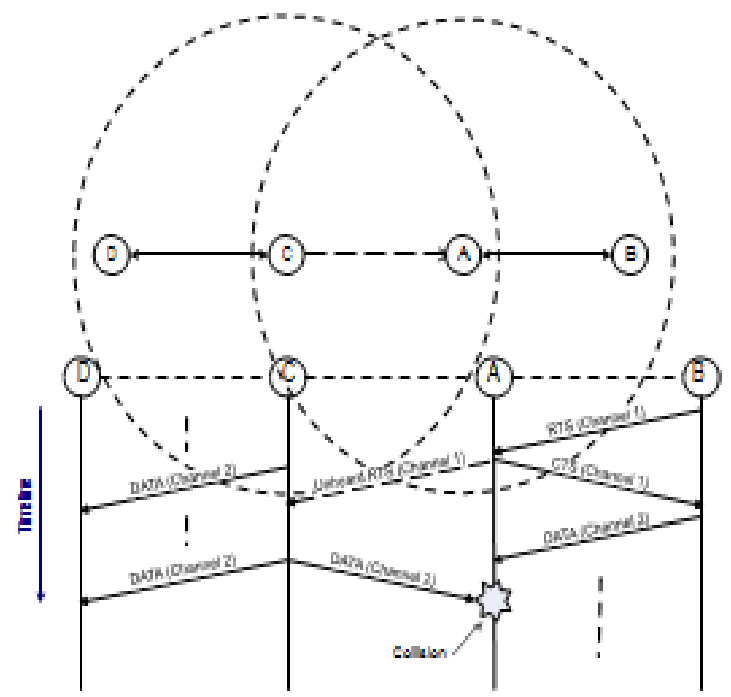

Fig 4 A scenario of multi-channel new hidden terminal in the multi-channel MAC protocols

Korakis et al. [19] proposed a circular MAC RTS, consisting of multiple RTS frames which are transmitted in a sequential manner on all the beams around the transmitter scanned area for dealing with deafness and hidden terminal problem a proper location table for each node, where the node finds the address receiver for dealing with these problems. This mechanism offers improvement over Omni directional transmissions, apart from it control packets which are in excess caused due to circular directional RTS will degrading the performance. Jakllari et al.[20] proposed a protocol of circular RTS and CTS MAC, consist of multiple directional CTS frames, which are transmitted in a circular fashion for handling deafness on the receiver side. Delay was increased in circular transmission results in large control overhead. Gossain et al.[21] proposed a protocol of MAC mainly for directional antennas consisting of multiple directional RTS and CTS messages which are being transmitted in a simultaneous manner through the beams of antenna having diametrically opposite directions with their neighbors. For achieving ongoing transmission of neighbors, they require RTS and CTS messages in a circular transmission for each data packet which is being transmitted and can incur large control overhead.

\section{SYSTEM DESCRIPTION AND OBJECTIVES}

In this we discuss this description with the antenna model with the structure of network. We will also discuss the basic objectives of WANET's with directional antennas.

\subsection{Network structure}

We have considered a WANET system , which basically consist of a number of wireless nodes which are equipped with directional antennas, as they are mobile and randomly distributed. We also assume that, mainly nodes are using symmetric radio channel which are basically homogenous in functionalities and capabilities.

The functioning of the network structure is shown in the diagram, where mainly the nodes in the network attempts for communications through MAC protocols. We can also see that, nodes communicate or trying to communicate with others mainly in directional mode. So in the scenarios, basically some nodes can endure the deafness and the new hidden terminal problems in the ongoing transmission of data period between node B and A.

\subsection{Antenna model}

Here, we consider that each node has a switched beam antenna which mainly comprises of $\mathrm{M}$ fixed beam patterns, shown in Fig 5. The antenna system operates on two separate modes, Omni and directional mode. In this Omni mode, a node mainly receives signals from all the directions with gain Go.Idle state node waits for the signals in Omni mode. In directional mode points on sensing signal the location of the direction of the antenna having signal power reaches in directional mode. Almost all research utilizes the same antenna model [18]. In CRDMAC protocol, the Omni mode is mainly utilized for receiving signals, and apart from it the directional mode is being utilized for data transmission and reception. Now we assume that the design in the directional mode is a sector in a circular fashion having constant sector gain with no gain outside.

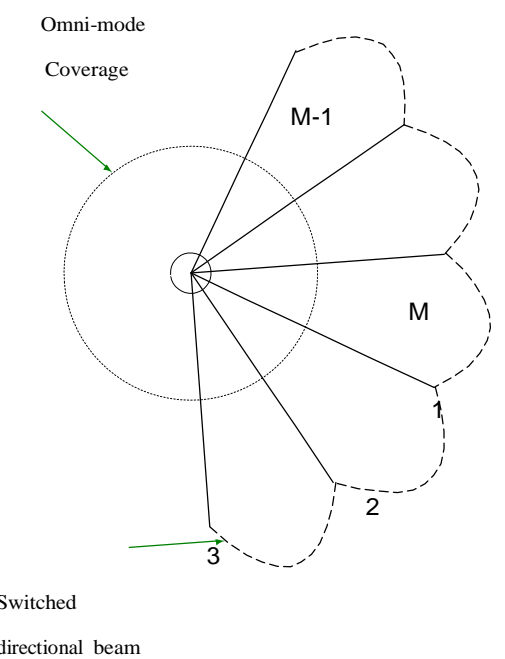

Fig 5 Antenna model with $M$ beams 
In directional mode, the antenna transmit a signal in any direction, by the use of an an array of elements. Individually Omni directional transmissions from these elements can interfere with each other, resulting in increase of strength in signal in many directions. Directional antennas with 1, 2, 4, 8, 16 etc. elements [6,26] are present .effectively Omni transmission having $\mathrm{M}$ sequentional directional transmissions, but when it has $\mathrm{M}$ antenna elements. An element which is important in the CRDMAC protocol mainly covers the maximum area around the transmitter with continuous sequential transmission.

\subsection{Objectives}

The deafness and the new hidden terminal problem which are caused in the basic DMAC and multichannel MAC protocols mainly waste the network capacity, and results in unfairness. But when multiple nodes attempt to communicate with same node, then the node that wins channel contention retain the privilege to use the channel for a long time. Apart from it the receiver remains busy all the time, the other transmitter nodes experiences unfairness. Mainly in this paper, we study the deafness problem (including the dead- lock problem) and new hidden terminal problem $\mathrm{s}$ in the DMAC protocols. Now we consider the conditions above in WANETs, and mainly focus on solving these problems with effective CRDMAC protocol for WANETs. problems caused in the basic DMAC and multi-channel MAC protocols wasting network capacity and resulting unfairness. But on the communication of multiple nodes with same node, channel contention node winning retains privilege for accessing channel for a much longer time. apart from it receiver is still busy for the maximum time and other nodes with transmitters have the experiences of unfairness. In this paper, we study the deafness problem (including the deadlock problem) and new hidden terminal problems in DMAC protocols. We consider the conditions above in WANETs, and focus on solving these problems with an effective CRDMAC protocol for WANETs.

\section{ACKNOWLEDGMENTS}

I am very grateful to Dr. Narendra singh yadav and Dr. Kavita ,for reviewing the manuscript in spite of their very busy schedule. They have made several important suggestions which enhanced this edition.

\section{CONCLUSION AND FUTURE WORK}

The objective of this paper is to have a detailed analysis for evaluating the performance of a mac protocol in directional antennas. We discussed in this paper various classification of directional antennas. We learned about DMAC and multichannel mac protocol. Then we have a overview of deafness and hidden terminal problem considering its various aspects. We also discuss the description of antenna model with the structure of network and the basic objectives of WANET's with directional antennas.

In future if loads were unpredicted or there are less number of competitive nodes we can make use of our proposed work for better efficiency and results. For the future we can use different randomization function which can change the behavior of the waiting and can mainly improve the performance of the network, and we can also use the Markov model and data mining algorithms for preparing the test that is directed to the network load and behave dynamically as per load and the need.

\section{REFERANCES}

[1] LU Huang1, LI Jie1*, DONG Zhongping2, JI Yusheng3.Design and Evaluation of CRDMAC: Circular RTR Directional MAC Protocol for WANETs.

[2] Vaishali Tiwari*, Prof. Damodar Tiwari. Prof. Vijay Lokhande. By Modifying BEB Algorithm an Optimized MAC 802.11 Protocols for Wireless Network .

[3] Er.Lipsa Walia ,Er.Pradeep Kumar Gaur, Dr. (Mrs.) Anupma Marwaha .RADNET Protocol enhancement by implementing RED routing algorithm using NS2 for Wireless Adhoc Networks

[4] TOH C K, Ad Hoc Mobile Wireless Networks: Protocols and Systems [M]. 1st ed. Prentice Hall, 2002.

[5] RUBINSTEIN M, MORAES I, CAMPISTA M, et al. A Survey on Wireless Ad Hoc Networks [J]. Mobile and Wireless Communication Networks, IFIP International Federation for Information Processing, 2006, 211: 1-33.

[6] DAI H, NG K-W, WU M-Y. An Overview of MAC Protocols with Directional Antennas in Wireless ad hoc Networks [C]// Proc. of Second International Conference on Wireless and Mobile Communications (ICWMC'06): Bucharest, Romania. IEEE Press, 2006:84.

[7] RAMANATHAN R, REDI J, SANTIVANEZ C, et al. Ad Hoc Networking With Directional Antennas: A Complete System Solution [J]. IEEE Journal on Selected Areas in Communications, 2005, 23(3): 496-506.

[8] IEEE 802.11, Wireless LAN medium access control (MAC) and physical layer (PHY) specifications, IEEE Standard [S] 1999.

[9] KO Y-B, CHOI J-M, VAIDYA N H. MAC protocols using directional antennas in IEEE802.11 based ad hoc networks [J]. Wireless Communications and Mobile Computing, 2008, 8(6): 783-795.

[10] CHOUDHURY R R, YANG X, RAMANATHAN R, et al. Using directional antennas for medium access control in ad hoc networks $[\mathrm{C}] / /$ Proc. of the 8th annual international conference on Mobile computing and networking (MobiCom'02): Atlanta, USA. ACM Press, 2002: 59-70.

[11] TAKAI M, MARTIN J, BAGRODIA R, et al. Directional virtual carrier sensing for directional antennas in mobile ad hoc networks [C]// Proc. of the 3rd ACM International Symposium on Mobile Ad Hoc Networking and Computing (Mo- biHoc'02): Lausanne, Switzerland. ACM Press, 2002: 183-193.

[12] KUMAR S, RAGHAVAN V S, DENG J. Medium Access Control protocols for ad hoc wireless networks: A survey [J]. Ad Hoc Networks. 2006, 4(3): 326-358.

[13] CHOUDHURY R, VAIDYA N H. Deafness: a MAC problem in ad hoc networks when using directional antennas $[C] / /$ Proc. of the 12th IEEE International Conference onNetwork Protocols (ICNP2004): Berlin, Germany. IEEE Press, 2004: 283-292.

[14] KO Y-B, SHANKARKUMAR V, VAIDYANH. Medium access control protocols using directional antennas in ad hoc networks [C]// Proc. of IEEE 9th Annual Joint Conference Of the IEEE Computer and Communications Societies 
(INFOCOM'00): Tel-Aviv, Israel IEEE Press, 2000, 1: $13-21$.

[15] SO J, VAIDYA N H. Multi-Channel MAC for Ad Hoc Networks: Handling Multi-Channel Hidden Terminals Using A Single Transceiver [C]// Proc. of the 5th ACM International Symposium on Mobile Ad Hoc Networking And Computing (MobiHoc'04): Tokyo, Japan. ACM Press, 2004: 222-233.

[16] JURDAK R, LOPES C, BALDI P. A survey, classification and comparative analysis of medium access control protocols for ad hoc networks [J]. IEEE Communications Surveys \& Tutorials. 2004, 6(1): 216.

[17] LEHNE P H, PETTERSEN M. An overview of smart antenna technology for mobile communications systems [J]. IEEE Communications Surveys \& Tutorials. 1999, 2(4): 2-13.

[18] BAZAN O, JASEEMUDDIN M. A SurveyOn MAC Proto- cols for Wireless Adhoc Networks with Beam forming Antennas [J]. IEEE Communications Surveys \& Tutorials2012, 14(2): 216-239.

[19] KORAKIS T, JAKLLARI G, TASSIULAS L. CDRMAC: A Protocol for Full Exploitation of Directional Antennas in Ad Hoc Wireless Networks [J]. IEEE Transactions on Mobile Computing. 2008, 7(2): 145-155.

[20] JAKLLARI G, BROUSTIS I, KORAKIS T, et al. Handling asymmetry in gain in directional antenna equipped ad hoc networks [C]// Proc. of IEEE 16th International Symposium on Personal, Indoor and Mobile Radio Communications (PIMRC'05): Berlin, Germany. IEEE Press, 2005, 2: 1284 - 1288.
[21] GOSSAIN H, CORDEIRO C, AGRAWAL D. MDA: an efficient directional MAC scheme For wireless ad hoc networks $[\mathrm{C}] / /$ Proc. of IEEE Global Telecommunications IEEE Press, 2005: 1-5.

[22] LEI Hongjiang, GAO Chao, GUO Yongcai, et al. Survey of multi-channel MAC protocols for IEEE 802.11-based wireless Mesh networks [J].The Journal of China Universities of Posts and Telecommunications. 2011, 18(2): 33-44.

[23] WANG J-P, ABOLHASAN M, SAFAEI F, et al. A survey on control separation techniques in multi-radio multichannel MAC protocols [C]// Proc. of International Symposium Communications and Information Technologies (ISCIT'07): Sydney, Australia. IEEE Press, 2007: 854-859.

[24] LU H, LI J, DONG Z, et al. CRDMAC: An Effective Circular RTR Directional MAC Protocol for Wireless Ad Hoc Networks [C]// Proc. of 2011 Seventh International Conference on Mobile Ad-hoc and Sensor Networks (MSN2011): Beijing, China. IEEE Press, 2011: 231-237.

[25] TOBAGI F, KLEINROCK L. Packet Switching in Radio Channels: Part II-The Hidden Terminal Problem in Carrier Sense Multiple-Access and the Busy-Tone Solution [J]. IEEE Transactions on Communications. 1975, 23(12): 14171433.

[26] TAKATA M, BANDAI M, WATANABE T. Performance Analysis of a Directional MAC Protocol for Location Information Staleness in MANETs [J]. IPSJ Digital Courier. 2005, 1: 518-527.

[27] BALANIS C A. Antenna Theory: Analysis and Design [M]. 3rd ed. Wiley-Interscience, 2005.

[28] Java Runtime Environment. Java, Sun Microsystems, Oracle Corporation [CP/OL]. http://www.java.com/. 